\title{
CORRIGENDUM
}

\section{Dietary carbohydrates, glycaemic load, food groups and newly detected type 2 diabetes among urban Asian Indian population in Chennai, India (Chennai Urban Rural Epidemiology Study 59) - CORRIGENDUM}

Viswanathan Mohan, Ganesan Radhika, Rangaswamy Mohan Sathya, Selvi Ramjothi Tamil, Anbazhagan Ganesan and Vasudevan Sudha doi:10.1017/S0007114509990468, Published by Cambridge University Press, 9 July 2009.

The authors noted that there is a small typographical error in Table 4 of this article.

The value for the 'percentage of newly diagnosed T2DM' in the 4th quartile of Glycemic Load should be $11.7 \%$ and not ' $2.6 \%$ '. The corrected table is supplied below.

Table 4. Risk for newly diagnosed type 2 diabetes according to carbohydrate, glycaemic load and dietary fibre*

(Unadjusted and adjusted odds ratios and $95 \%$ confidence intervals)

\begin{tabular}{|c|c|c|c|c|c|c|c|c|c|}
\hline \multirow[b]{3}{*}{ Variable } & \multicolumn{8}{|c|}{ Quartiles } & \multirow[b]{3}{*}{$P$ for trend $\dagger$} \\
\hline & \multicolumn{2}{|c|}{1 (Lowest) (n 460) } & \multicolumn{2}{|c|}{$2(n 461)$} & \multicolumn{2}{|c|}{$3(n 461)$} & \multicolumn{2}{|c|}{4 (Highest) (n 461) } & \\
\hline & OR & $95 \% \mathrm{Cl}$ & OR & $95 \% \mathrm{Cl}$ & OR & $95 \% \mathrm{Cl}$ & OR & $95 \% \mathrm{Cl}$ & \\
\hline \multicolumn{10}{|l|}{ Carbohydrates $(\mathrm{g} / \mathrm{d}) \ddagger$} \\
\hline Range & \multicolumn{2}{|c|}{$153 \cdot 5-333 \cdot 7$} & \multicolumn{2}{|c|}{$>333 \cdot 7-403 \cdot 4$} & \multicolumn{2}{|c|}{$>403 \cdot 4-498 \cdot 4$} & \multicolumn{2}{|c|}{$>498 \cdot 4-763 \cdot 2$} & \\
\hline Median & \multicolumn{2}{|c|}{$294 \cdot 0$} & \multicolumn{2}{|c|}{368.9} & \multicolumn{2}{|c|}{443.4} & \multicolumn{2}{|c|}{$587 \cdot 1$} & \\
\hline Newly diagnosed T2DM & & & & & & & & & $<0.001$ \\
\hline$n$ & \multicolumn{2}{|r|}{20} & \multicolumn{2}{|r|}{36} & \multicolumn{2}{|r|}{44} & \multicolumn{2}{|c|}{56} & \\
\hline$\%$ & \multicolumn{2}{|r|}{$4 \cdot 1$} & \multicolumn{2}{|c|}{$7 \cdot 8$} & \multicolumn{2}{|r|}{$9 \cdot 1$} & \multicolumn{2}{|c|}{$13 \cdot 2$} & \\
\hline Unadjusted OR for diabetes & 1.00 & Reference & 1.97 & $1.13,3.46$ & $2 \cdot 41$ & $1 \cdot 39,4 \cdot 16$ & 3.59 & $2 \cdot 12,6 \cdot 09$ & $<0.001$ \\
\hline Adjusted OR for diabetes & 1.00 & Reference & 3.05 & $1.62,5.77$ & 3.53 & $1.89,6.56$ & 4.55 & $2.49,8.29$ & $<0.001$ \\
\hline \multicolumn{10}{|l|}{ Glycaemic index $\ddagger$} \\
\hline Range & \multicolumn{2}{|c|}{$57 \cdot 8-67 \cdot 1$} & \multicolumn{2}{|c|}{$>67.1-68.9$} & \multicolumn{2}{|c|}{$>68.9-70.3$} & \multicolumn{2}{|c|}{$>70 \cdot 3-73.9$} & \\
\hline Median & \multicolumn{2}{|c|}{$65 \cdot 4$} & \multicolumn{2}{|c|}{$68 \cdot 0$} & & $69 \cdot 5$ & & 1.3 & \\
\hline Newly diagnosed T2DM & & & & & & & & & $<0.001$ \\
\hline$n$ & & 25 & & 33 & & 48 & & 50 & \\
\hline$\%$ & & $5 \cdot 2$ & & $7 \cdot 4$ & & $11 \cdot 1$ & & 0.4 & \\
\hline Unadjusted OR for diabetes & 1.00 & Reference & 1.91 & $0.68,2.08$ & 1.82 & $1.07,3.09$ & $2 \cdot 01$ & $1 \cdot 19,3.40$ & 0.020 \\
\hline Adjusted OR for diabetes & 1.00 & Reference & 1.38 & $0.76,2.51$ & $2 \cdot 12$ & $1.20,3.75$ & $2 \cdot 51$ & $1.42,4.43$ & 0.006 \\
\hline Glycaemic load $\ddagger$ & & & & & & & & & \\
\hline Range & & $9-226 \cdot 4$ & $>2$ & $\cdot 4-276 \cdot 6$ & $>2$ & $6-344.6$ & & $6-540 \cdot 8$ & \\
\hline Median & & 98.7 & & $52 \cdot 2$ & & $307 \cdot 2$ & & 13.4 & \\
\hline Newly diagnosed T2DM & & & & & & & & & $<0.001$ \\
\hline$n$ & & 17 & & 37 & & 48 & & 54 & \\
\hline$\%$ & & 3.5 & & 7.9 & & $10 \cdot 3$ & & 1.7 & \\
\hline Unadjusted OR for diabetes & 1.00 & Reference & $2 \cdot 33$ & $1 \cdot 29,4 \cdot 21$ & $3 \cdot 12$ & $1.77,5.51$ & 3.91 & $2 \cdot 23,6 \cdot 85$ & $<0.001$ \\
\hline Adjusted OR for diabetes & 1.00 & Reference & 2.69 & $1.42,5.09$ & 3.85 & $2 \cdot 08,7 \cdot 10$ & 4.25 & $2 \cdot 33,7.77$ & $<0.001$ \\
\hline
\end{tabular}


Table 4. Continued

\begin{tabular}{|c|c|c|c|c|c|c|c|c|c|}
\hline \multirow[b]{3}{*}{ Variable } & \multicolumn{8}{|c|}{ Quartiles } & \multirow[b]{3}{*}{$P$ for trend $\dagger$} \\
\hline & \multicolumn{2}{|c|}{1 (Lowest) ( $n$ 460) } & \multicolumn{2}{|c|}{$2(n 461)$} & \multicolumn{2}{|c|}{$3(n 461)$} & \multicolumn{2}{|c|}{4 (Highest) (n 461) } & \\
\hline & OR & $95 \% \mathrm{Cl}$ & OR & $95 \% \mathrm{Cl}$ & OR & $95 \% \mathrm{Cl}$ & OR & $95 \% \mathrm{Cl}$ & \\
\hline \multicolumn{10}{|l|}{ Dietary fibre $(\mathrm{g} / 1000 \mathrm{~kJ})$} \\
\hline Range & \multicolumn{2}{|c|}{$0.9-2.5$} & \multicolumn{2}{|c|}{$>2.5-2.9$} & \multicolumn{2}{|c|}{$>2.9-3.3$} & \multicolumn{2}{|c|}{$>3.3-7.2$} & \\
\hline Median & \multicolumn{2}{|c|}{$2 \cdot 1$} & \multicolumn{2}{|c|}{$2 \cdot 8$} & \multicolumn{2}{|c|}{$3 \cdot 1$} & \multicolumn{2}{|c|}{$3 \cdot 7$} & \\
\hline Newly diagnosed T2DM & & & & & & & & & $<0.001$ \\
\hline$n$ & \multicolumn{2}{|c|}{42} & \multicolumn{2}{|c|}{66} & \multirow{2}{*}{\multicolumn{2}{|c|}{$\begin{array}{l}37 \\
8.0\end{array}$}} & \multirow{2}{*}{\multicolumn{2}{|c|}{$\begin{array}{c}11 \\
2.4\end{array}$}} & \\
\hline$\%$ & \multicolumn{2}{|c|}{$9 \cdot 1$} & \multicolumn{2}{|c|}{$14 \cdot 3$} & & & & & \\
\hline Unadjusted OR for diabetes & 1.00 & Reference & 1.66 & $1 \cdot 10,2.51$ & 0.87 & $0.56,1.38$ & 0.24 & $0.13,0.48$ & $<0.001$ \\
\hline Adjusted OR for diabetes & 1.00 & Reference & 1.95 & $1.24,3.06$ & $1 \cdot 19$ & $0.73,1.95$ & 0.31 & $0.15,0.62$ & $<0.001$ \\
\hline
\end{tabular}

T2DM, type 2 diabetes mellitus.

*The adjusted model controlled for age (years in quintiles), sex (males, females), BMI (continuous), family history of diabetes (three categories), cigarette smoking (categorised as non-smokers and habitual smokers), alcohol (never, past and current consumers), physical activity (strenuous, no exercise, sedentary) and income in Indian rupees $(<2000,2000-5000,>5000-10000,>10000)$. There was additional adjustment for dietary fibre (for the carbohydrate and glycaemic load models) and for carbohydrate (for the fibre model).

†Tests for linear trend were conducted across increasing categories by treating the medians of intake in categories as continuous variables.

$\ddagger$ Energy adjusted using the residual method.

\section{Reference}

1. Mohan V, Radhika G, Sathya RM, et al. (2009) Dietary carbohydrates, glycaemic load, food groups and newly detected type 2 diabetes among urban Asian Indian population in Chennai, India (Chennai Urban Rural Epidemiology Study 59). Br J Nutr 102, 1498-1506. Published by Cambridge University Press, 9 July 2009, doi:10.1017/S0007114509990468. 